\title{
A Novel Model for Prediction of RNA binding Proteins
}

\author{
Shingo Kikugawa ${ }^{1}$, Hideki Takehara ${ }^{2}$, Satoru Kuhara ${ }^{2}$, \\ and Makoto Kimura ${ }^{1 *}$ \\ ${ }^{1}$ Laboratory of Biochemistry, Department of Bioscience and \\ Biotechnology, Faculty of Agriculture, Graduate School, \\ Kyushu University, Fukuoka 812-8581, Japan \\ ${ }^{2}$ Laboratory of Molecular Gene Technics, Faculty of Agriculture, \\ Graduate School, Kyushu University, Fukuoka 812-8581, Japan \\ *E-mail: mkimura@agr.kyushu-u.ac.jp
}

(Received December 3, 2004; accepted January 13, 2005; published online February 4, 2005)

\begin{abstract}
We have developed an efficient prediction procedure for RNA binding proteins with oligosaccharide/oligonucleotide binding-fold (OB-fold). First, all pairwise superimpositions of 96 OB-fold structures included in Structural Classification of Proteins (SCOP) database classified them into four distinct groups on the basis of structural similarity. The proteins belonging to each group were divided into RNA binding proteins and non-RNA binding proteins. The structure-based sequence alignment of RNA binding proteins in each group were made to build profile hidden Markov models (HMMs). The reliability of HMMs thus obtained was first evaluated by the application to the PDB40 sequence dataset; RNA binding proteins with OB-fold classified into OB-fold in SCOP were selected, giving E-values less than 1.0. The next application of HMMs to sequence database of the hyperthermophilic archaeon Pyrococcus horikoshii OT3 detected several RNA binding proteins, including tRNA synthetases, initiation factor, transcriptional regulatory proteins, and ribosomal protein $\mathrm{L10E}$ as RNA binding proteins with OB-fold. These results suggested that HMM derived from this study has information about RNA binding proteins with OB-fold. The present analysis strongly suggested 4 hypothetical proteins in $P$. horikoshii to be RNA binding proteins with OB-fold. Furthermore, the application of the present model to the rice full-length cDNA sequence database suggested 14 hypothetical proteins to be RNA binding proteins with OB-fold. It is known that some of the motifs have no specific biological function alone but are part of larger structural and functional assembles. Thus, the present method would provide clues as to protein functions of unannotated proteins and also be useful for a target selection for structural genomics.
\end{abstract}

Key Words: hidden Markov models, OB-fold, protein function prediction, Pyrococcus horikoshii, RNA binding protein

Area of Interest: Bioinformatics and Bio Computing 


\section{Introduction}

The genome sequencing efforts have now provided biologists with coding information for thousands of new proteins, most of which have no known function that can be predicted using sequence-based methods [1]. Hence, assigning functions to novel proteins is one of the most important problems in the postgenomic era. Recent advances in structural analysis, together with a known fact that three-dimensional structures are conserved across a much greater evolutional distance than recognizable primary sequences, have led to the concept of "structural genomics", the determination of three-dimensional protein structures on a genome-wide scale [2]. An important use of three-dimensional protein structural information of proteins is to uncover clues as to protein functions that are not detectable from sequence analysis [3][4]. Although experimental structure determination methods are providing high-resolution structure information about a subset of the proteins [5], computational methods for prediction of protein function are required for large fraction of sequences whose structures will not be determined experimentally.

Recently, several groups have developed algorithms to predict protein function employing sequence-based comparisons. A simple and widely used strategy is the identification of a high sequence similarity between proteins of known and unknown function that is then used to transfer the specific function [6][7][8]. However, lower levels of sequence similarity can only be used to transfer general functions and therefore, this approach is not reliable. On the other hand, non-sequence-based approaches for the protein functional prediction, including the analysis of gene expression patterns, phylogenetic profiles, protein fusions, and protein-protein interactions have been reported. Clustering analysis of gene expression data can be used to predict functions of unannotated proteins based on the idea that coexpressed genes are more likely to have similar functions [9][10]. In addition, functional predictions have been modeled as pattern recognition problems based on sequence homologies and structural information as well as phenotype data [11][12][13]. Although they have been extensively improved, an alternative computational method should be required for high-throughput prediction of the protein functions. As for the protein structural prediction, there have been a number of promising advances in de novo structure prediction [14][15]. A particularly successful method, called Rosetta, uses information from the Protein Data Bank (PDB) to estimate possible conformation for local sequence segments [16][17]. Rosetta was recently used to generate both fold and function predictions for Pfam protein families [18] and Halobacterium sp. NRC-1 [19] and provide general function information for many proteins of unknown function. In spite of these successes, the accuracy of de novo methods is still known to be problematic.

There is growing evidence that RNA molecules play essential roles in biological processes of living cells, such as pre-mRNA splicing in the spliceosome [20] and peptide-bond formation in the ribosome [21]. RNA molecules usually perform these functions in close association with RNA binding proteins, and thus RNA binding proteins play crucial roles in a wide range of biological processes. To date, a large number of structural information about RNA binding proteins has become available, and their knowledge has contributed to full understanding of the biological role of these proteins. In addition, these studies revealed that they share structural motifs, such as oligonucleotide/oligosaccharide binding fold (OB-fold), ribonucleoprotein (RNP), double-stranded RNA binding domain (dsRBD), K homology (KH) and helix-turn-helix motif [22]. During the course of studies on three-dimensional structures of ribosomal proteins, it has been known that many ribosomal proteins share structural motifs, even though the structural resemblance is not reflected in an obvious homology at the sequence level [23]. In our own studies, it was found that ribosomal proteins S1, S12, S17, and L2 fold into OB-fold [24], while S6, L5, and L30 share RNP fold [25], though they share few residues in the corresponding domains. These findings have led us 
to the expectation that common features occurred in the structural motifs would be invaluable information for non sequence-based prediction of RNA binding proteins.

In this paper, we describe the building of novel hidden Markov models (HMMs) which were derived from structure-based sequence alignment of RNA binding proteins with OB-fold. Their validity was evaluated and discussed by application to PDB40, Pyrococcus horikoshii OT3, and rice full length cDNA sequence databases.

\section{Materials and methods}

\subsection{Collection of OB-fold structures}

Protein structures with OB-fold were taken from the protein structures belonging to the OB-fold in Structural Classification of Proteins (SCOP) database (release 1.61) [26]. When the structural domains with OB-fold were not notified in the proteins classified into the OB-fold in the SCOP, we manually selected OB-fold domains on the criterion that a five-stranded antiparallel $\beta$-sheet forms a closed $\beta$-barrel. The atomic coordinates of the OB-folds were obtained from PDB [27]. In this collection, identical structures with a few mutations or in complex with different ligands were omitted from datasets.

\subsection{Rectifications of secondary structures}

The reliability of our structure alignment method with vector representation of secondary structures is sensitive for their definitions. DSSP [28] included in Sequential Structure Alignment Program (SSAP), which is the program to define secondary structures by the pattern of hydrogen bonding of amino acids in crystal structure coordinates, often gives an inconvenient definition for our structural alignment method because of its strictness. Thus, consecutive long twisting $\beta$-strands in OB-fold are defined as a few pieces of short $\beta$-strands. This misdefinition of the secondary structure would be caused by a low resolution analysis or disordered structure. When two $\beta$-strands in OB-fold form antiparallel $\beta$-sheet, and one $\beta$-strand forms an angle of more than $100^{\circ}$ with the other $\beta$-strand, we assumed that the two $\beta$-strands would be a single $\beta$-strand.

\subsection{Pairwise superimposition of OB-fold structures}

All combinations of pairwise superimposition of OB-folds were carried out with a vector alignment method of secondary structures, for which the program was written in $\mathrm{C}++$ language on RedHat Linux 8 for IBM PC/AT compatibles with $2.4 \mathrm{GHz}$ pentium4 processor to complete a huge number of superimposition combinations of OB-folds. The vector alignment method is an algorithm partially used at the initial search of the Dali 3D search server [29]. In brief, two pairs of structurally corresponding $\beta$-strands of OB-folds were determined by the result of SSAP [30] and transformed to align their structures by overlapping the equivalent $\beta$-strands. Structure alignment of a pair of candidates was performed with alignment of corresponding unit vectors along by each secondary structure of the OB-folds. All pairs of OB-folds were superimposed and their original coordinates were transformed to superimposed positions. Then, root-mean-square deviations (RMSD) derived from the superimpositions were calculated with the average distance between two $\mathrm{C} \alpha$ points of the SSAP determined equivalent pairs of residues. 


\subsection{Cluster analysis of OB-fold structures}

The cluster analysis with Ward's method [31] using "R 1.7" [32], a language and environment for statistical computing was performed to classify OB-fold structures based on the RMSDs obtained from the structure superimpositions.

\subsection{Structure-based multiple alignment}

The structure-based multiple sequence alignments were constructed by a heuristic method known as "star alignment method" [33]. Two amino acid sequences, a representative amino acid sequence whose structure has the least average RMSD at the structural superimposition and the other sequence belonging to the same groups, were arranged in two lines:

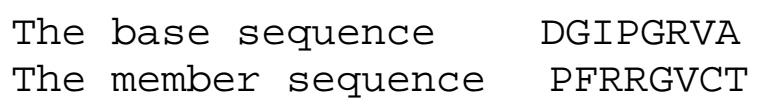

where the base and the member sequences indicate amino acid sequences of the representative structure and the other structure in the group, respectively. Equivalent residues of the two sequences were aligned by referring to the SSAP results. When a residue could not be aligned to any residues of the opponent sequence, a gap (-) was inserted into the opponent sequence. All pairwise alignments of the member sequences with the representative sequence were done in the same manner as that described above.

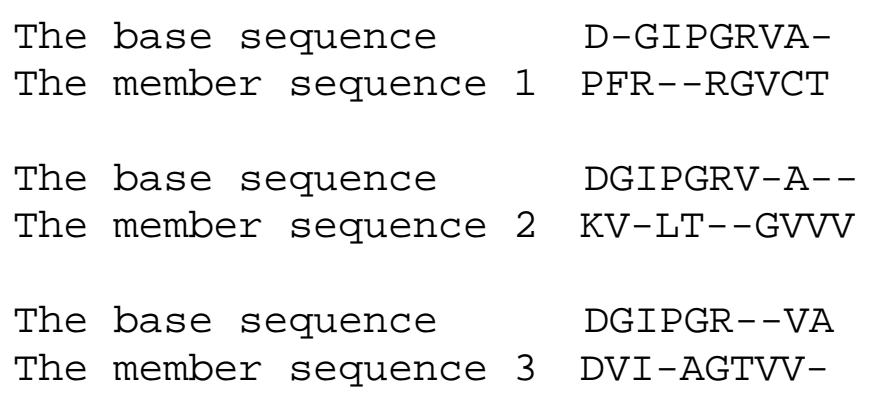

In this example, there are four members including the representative structure in a group. During pairwise structural alignments, several gaps were inserted at distinct positions in the representative sequences. Then, additional gaps were inserted into the representative sequence so as to give the identical base sequence with several gaps. Next, gaps were inserted into the member sequences so as to give the best alignment with corresponding residues.

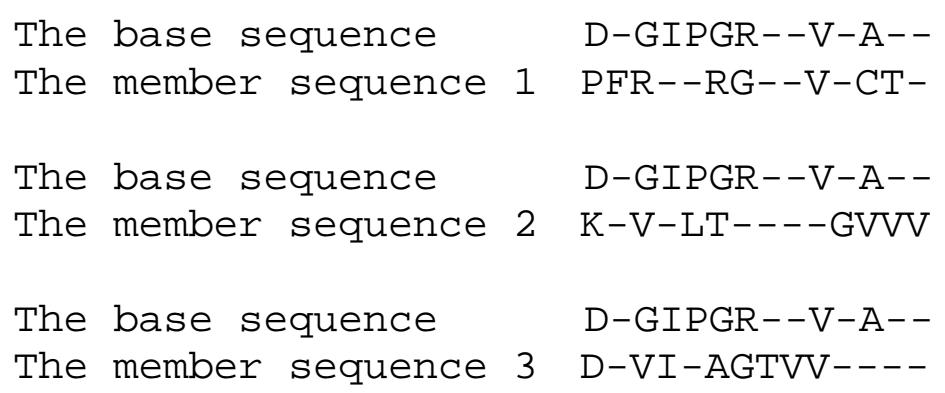



below.

Finally, a set of pairwise alignments was merged to provide a multiple alignment, as shown

$\begin{array}{lll}\text { The base alignment } & \text { D-GIPGR--V-A-- } \\ \text { The member sequence } 1 & \text { PFR--RG--V-CT- } \\ \text { The member sequence } 2 & \text { K-V-LT----GVVV } \\ \text { The member sequence } 3 & \text { D-VI-AGTVV---- }\end{array}$

\subsection{Building hidden Markov models and database search}

Profile hidden Markov models (HMMs) were built from the structure-based multiple alignments of OB-folds to extract common structural properties using the profile HMM software package HMMER [34]. The sequence database of PDB40 [35], a hyperthermophilic archaeon Pyrococcus horikoshii OT3 [36] and japonica rice full length cDNAs [37] were used for evaluation of HMMs. Databases were searched with an E-value described by HMMER [34].

\section{Results and discussion}

The five-stranded $\beta$-barrel motif designated as OB-fold was first observed in four different proteins which bind ologonucleotides or oligosaccharides: staphylococcal nuclease, anticodon binding domain of asp-tRNA synthetase and B-subunits of heat-labile enterotoxin and verotoxin-1 [38]. The common fold of the four proteins has a five-stranded $\beta$-sheet coiled to form a closed

A

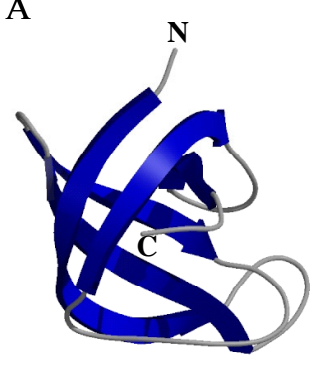

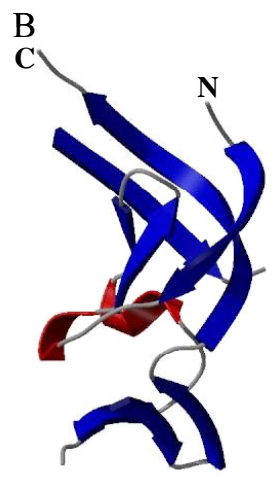

C

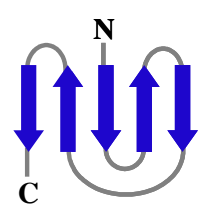

Figure 1. OB-fold structures.

A, OB-fold structure consists of a five-stranded antiparallel $\beta$-sheet with a Greek Key $\beta$ barrel typified by ribosomal protein S12 (1FJF). B, OB-folds occasionally have an insertion of $\alpha$ - or $3_{10}$-helix between $\beta$-strands and an additional structure consisting of two or three $\beta$-strands, as shown in the structure of RNA polymerase subunit RBP4 (1GO3). C, A topological diagram showing the secondary structure of OB-fold. Figures A and B were produced with the programs MOLSCRIPT [39] and Raster3D [40]. 


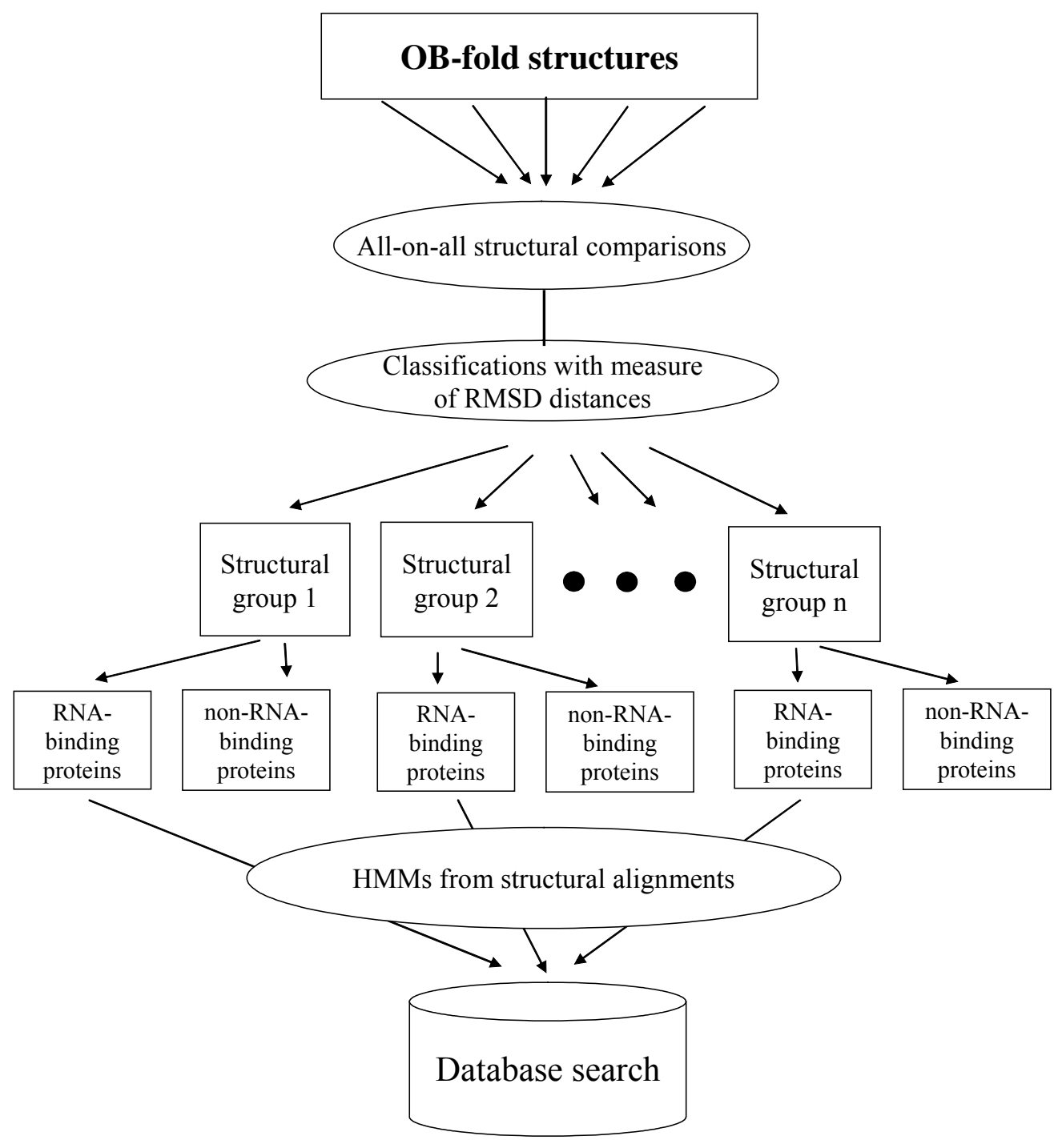

Figure 2. Strategy for developing a novel HMM for prediction of RNA binding proteins with OB-fold.

$\beta$-barrel (Figure 1). The barrel frameworks can be superimposed with RMSD of 1.4-2.2 $\AA$, though no similarities can be observed in the corresponding alignment of the four protein sequences. On the assumption that structural information occurred in OB-fold could be extracted from their structural alignments, we attempted to develop a novel computational method for prediction of RNA binding proteins by HMMs. A general strategy for building novel HMMs is presented in Figure 2.

\subsection{Superimposition of OB-fold structures}

First, protein structures with OB-fold were collected from SCOP database and their atomic coordinates were obtained from PDB, as described under Materials and methods. As a result, 84 proteins have OB-fold domains in which 72 proteins have a single OB-fold, whereas 12 proteins are comprised of two OB-folds. In total, 96 OB-fold structures were chosen for the present study (Table 1). Among 96 structures, 36 OB-fold structures are known to have potential to bind RNA. 
To gain an insight into a structural relationship, all possible pairwise superimpositions of OB-fold structures were done by using the vector alignment method, as described under Materials and methods. It took less than half second to superimpose a pair of OB-folds on the $2.4 \mathrm{GHz}$ pentium 4 processor excepting the calculation time of SSAP. The reliability of this method for vector alignments of secondary structures depends on definition of secondary structures and their lengths. Indeed, there were 124 small gaps between two consecutive $\beta$-strands in 96 OB-fold structures. As described under Materials and methods, when the angles between the two $\beta$-strands separated by small gaps were more than $100^{\circ}$, they were assumed to be a single $\beta$-strand. The results showed that the superimposition of OB-folds from inorganic pyrophosphatase (PDB: 1E9G) and initiation factor eIF2 $\alpha$ (1KL9) gave the least RMSD of $0.939 \AA$, whereas that of tissue inhibitor metalloproteinase (TIMP) (1BR9) and DNA ligase (1FVI) yielded the largest RMSD of 19.677 . Furthermore, OB-folds from TIMP - eIF2 $\alpha$, superantigen toxins (1BXT) - ribosomal protein L2 (1RL2), cold shock protein (1G6P) - RNA polymerase subunit RBP8 (1I5O), and molybdate binding protein (1GUT) - bacterial AB5 toxins (1TII) could not be superimposed because of their

Table 1. A list of OB-fold structures used in this study.

OB-folds were searched from SCOP and structure files were obtained from PDB.

\begin{tabular}{|c|c|c|c|}
\hline \multicolumn{4}{|l|}{$\overline{\text { RNA binding Proteins }}$} \\
\hline Initiation Factor & 1C0A:A:10-106 & 1FJF:Q:2-105 & RNA Polymerase II \\
\hline $1 \mathrm{AH} 9:: 6-70$ & 1EFW:A:10-104 & $1 J J 2: A: 35-84$ & 1GO3:E:80-167 \\
\hline 1BKB::75-139 & 1KRS::65-149 & 1RIP::8-64 & $1150: \mathrm{H}: 2-62$ \\
\hline 1D7Q:A:41-109 & 1LYL:A:63-150 & 1RL2:A:71-117 & 1I50:H:92-146 \\
\hline 1JT8:A:19-86 & Cold Shock Protein & S1 RNA-binding domain & mRNA Capping Enzyme \\
\hline 2EIF:A:74-132 & 1C9O:A:1-66 & 1HH2:P:133-198 & 1CKM:A:240-300 \\
\hline 1KL9:A:14-87 & $1 C S P:: 1-67$ & 1K0R:A:110-183 & RHO Termination Factor \\
\hline 1LUZ:A:10-88 & 1G6P:A:1-66 & 1SRO::1-76 & $1 \mathrm{~A} 62:: 46-125$ \\
\hline 1JJG:A:31-102 & 1H95:A:9-76 & Myf domain & Staphylococcal Nuclease \\
\hline tRNA Synthetase & $1 \mathrm{MJC}:: 2-70$ & 1EUJ:A:7-96 & 1SNC::7-96 \\
\hline 1ASZ:A:105-200 & Ribosomal Protein & 1GD7:A:10-90 & \\
\hline 1B8A:A:14-100 & 1FJF:L:31-105 & 1PYS:B:41-118 & \\
\hline \multicolumn{4}{|l|}{ non RNA binding Proteins } \\
\hline ssDNA-binding Protein & 1BXT:A:30-117 & 3CHB:D:12-103 & DNA Helicase \\
\hline $1 G P C:: 38-195$ & 1ENF:A:23-105 & Molybdate-binding Protein & 1BVS:A:1-64 \\
\hline 1GVP::1-65 & 1ESF:A:31-120 & 1B9M:A:127-183 & 1CUK::1-64 \\
\hline 1JE5:A:3-181 & 1ET9:A:18-95 & 1B9M:A:200-252 & Metalloproteinase Inhibitor \\
\hline 1JMC:A:193-292 & 1EU3:A:15-95 & 1FR3:A:1-67 & 1BQQ:T:16-109 \\
\hline 1JMC:A:311-403 & 1JCK:B:30-121 & $1 G 29: 1: 241-301$ & 1BR9::15-109 \\
\hline 1KXL:A:23-147 & $1 \mathrm{STE}:: 28-120$ & $1 G 29: 1: 302-358$ & 1UEA:B:15-107 \\
\hline 1OTC:A:51-150 & 2QIL:A:15-96 & 1GUT:A:2-68 & Chemotaxis Protein CheW \\
\hline 1OTC:A:223-300 & 3 SEB::30-122 & 1H9M:A:1-73 & 1K0S:A:30-95 \\
\hline 1OTC:A:365-495 & Superantigen-like Protein & 1H9M:A:74-141 & Histidine Kinase CheA \\
\hline 1OTC:B:37-129 & 1M4V:A:23-100 & Inorganic Pyrophosphatase & 1B3Q:A:561-626 \\
\hline 1PFS:A:1-73 & Bacterial AB5 Toxin & 1E9G:A:90-162 & Laminin-binding Domain of Agrin \\
\hline 1QUQ:A:68-151 & 1EFI:D:12-103 & 1I40:A:10-112 & 1JB3:A:14-110 \\
\hline 1QUQ:B:21-90 & 1PRT:B:99-193 & 1QEZ:A:1010-1110 & RecG \\
\hline 1QVC:A:1-115 & 1PRT:D:1-110 & 2PRD::10-112 & 1GM5:A:165-250 \\
\hline 3ULL:A:10-124 & 1PRT:F:2-99 & DNA Ligase & Tail-associated Lysozyme gp5 \\
\hline Bacterial Superantigens & 1QNU:A:101-169 & $1 \mathrm{~A} 0 \mathrm{I}:: 240-331$ & 1K28:A:6-129 \\
\hline $1 \mathrm{AN} 8:: 20-95$ & 1TII:D:14-98 & 1DGS:A:315-392 & \\
\hline 1B1Z:A:28-107 & 2BOS:A:102-169 & 1FVI:A:190-293 & \\
\hline
\end{tabular}

dissimilarity. During the process of superimpositions, the ribosomal protein S17 from Bacillus stearothermophilus [41] (1RIP) was unable to be superimposed on other structures. The 
reinterpretation of the secondary structure of S17 with DSSP revealed a slight distinct topology from other OB-folds. Hence, no superimposition was further carried out on the protein S17.

\subsection{Cluster analysis of OB-fold structures}

The cluster analysis of OB-fold structures was done with Ward's method based on the RMSD distances obtained from their overall superimpositions. The RMSD distant dendrogram derived from the cluster analysis is shown in Figure 3. It became clear that OB-folds are classified into four structural groups at 20 rescaled distance cluster combine. The OB-fold structures belonging to groups $1-4$ are given in Table 2. Proteins belonging to the groups 1 and 2 are predominantly composed of $\beta$-strands; those included in the group 1 typically have five-stranded anti-parallel $\beta$-sheet, while those in the group 2 have additional short $\beta$-strands or some insertional residues between $\beta 3$ and $\beta 4$ strands. Proteins belonging to the groups 3 and 4 have additional $\alpha$-helix between $\beta 3$ and $\beta 4$ strands, while $\beta 1$ in the group 3 is rather shorter than the corresponding $\beta$-strands in the other groups. The group 1 includes 23 proteins, such as initiation factors (aIF5a, eIF1a), Myf domain, single-stranded DNA binding proteins, and molybdate-binding proteins. Among them, 9 proteins are known to be RNA binding proteins. In the group 2, there are 30 proteins including 20 RNA binding proteins. Ribosomal proteins (S1, S12, S17 and L2), cold shock proteins, initiation factors (IF1, aIF1a, eIF2a), inorganic pyrophosphatase and other 6 kinds of proteins belong to the group 2 . The group 3 predominantly includes non-RNA binding proteins, such as eight superantigens (toxic proteins), while the group 4 has 6 tRNA-synthetases (RNA binding proteins) and 28 non-RNA binding proteins, such as SSBs, bacterial B5 toxins and other 5 proteins.

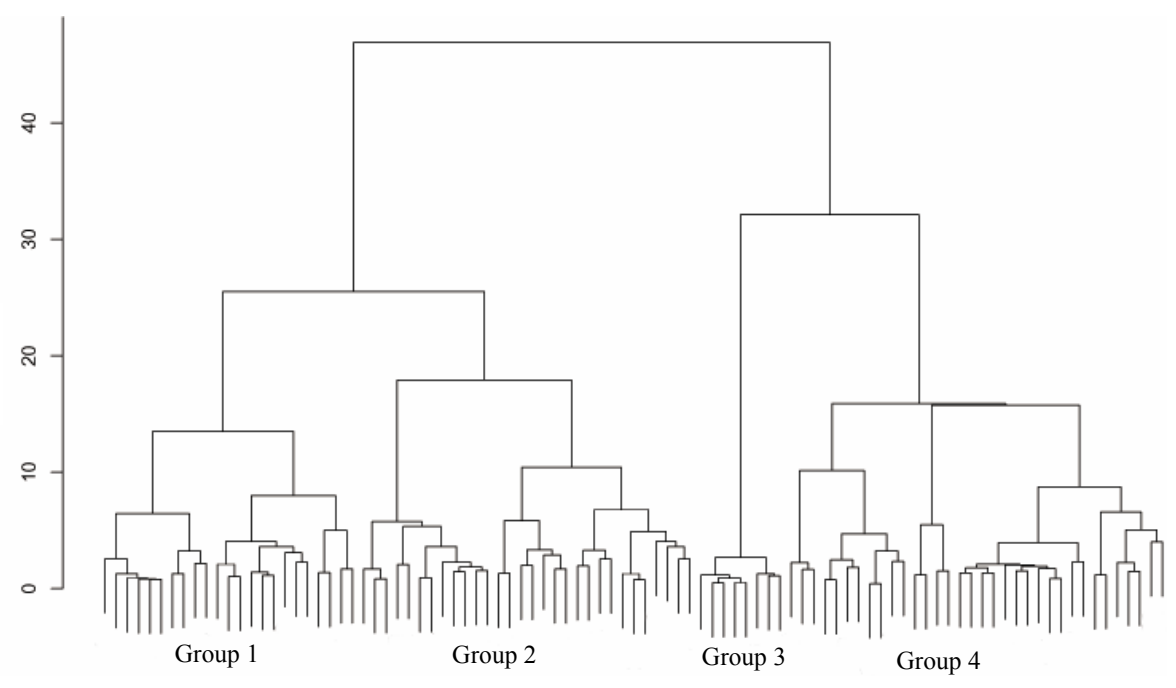

Figure 3. Dendrogram of OB-fold structures.

The dendrogram was drawn from the cluster analysis of OB-fold structures. The result shows that OB-fold structures are classified into four structural groups. 


\subsection{Structure-based multiple alignment}

The amino acid sequences of RNA binding proteins or non-RNA binding proteins belonging to the same OB-fold groups were aligned on the basis of secondary structure, as described under Materials and methods.

The structure-based multiple alignments of RNA-binding members in the each group are shown in Figure 4. The average RMSD distances among residues in alignment columns were calculated with the superimposed structure files. In general, the amino acids on $\beta$-strands provide short RMSD distances, indicating that the $\beta$-strand structures are conserved among OB-folds. Although we manually attempted to find common properties in amino acid sequences of RNA binding proteins belonging to the same groups, general features occurred in the amino acid sequences remained obscure.

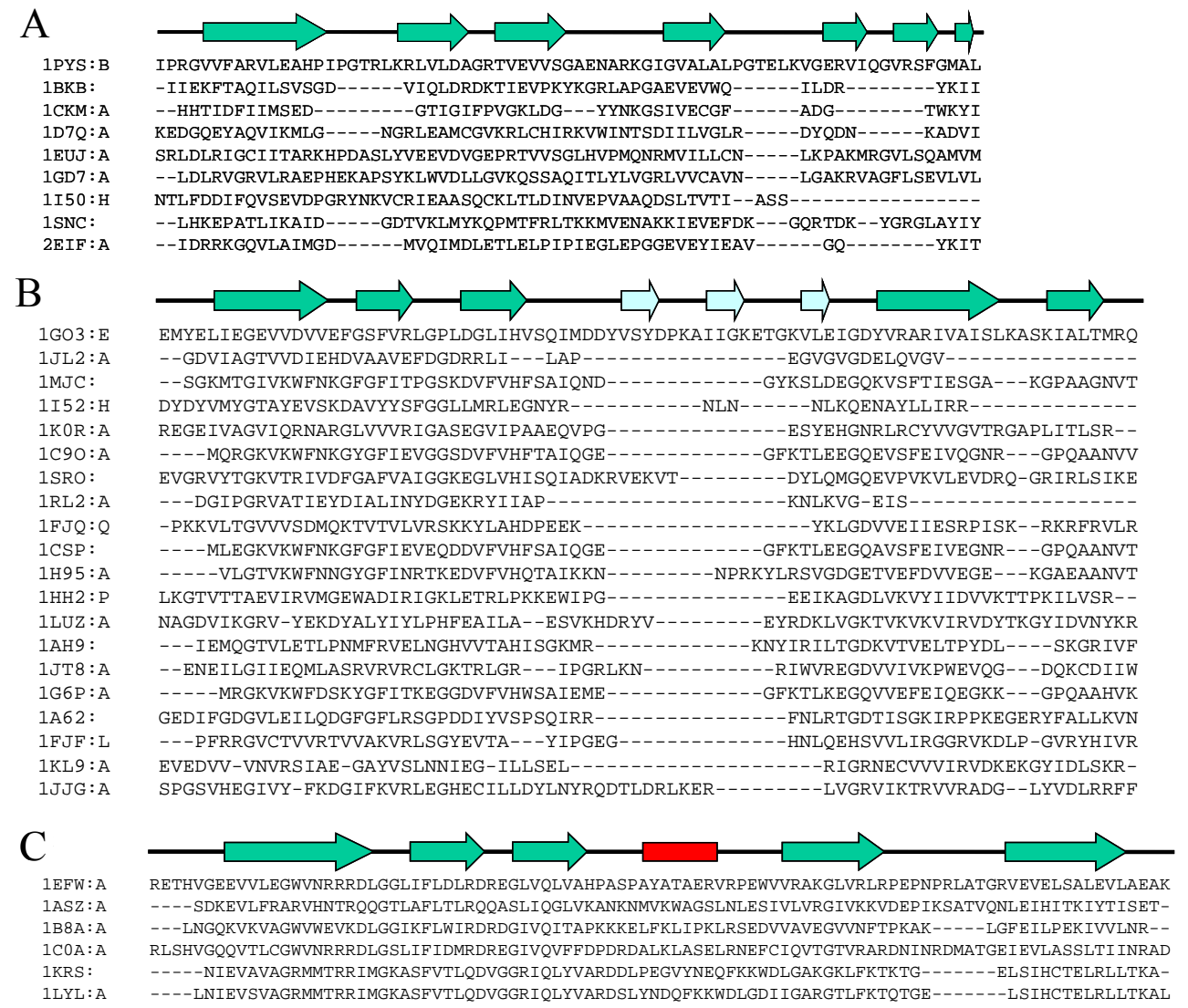

Figure 4. Structure-based multiple sequence alignments of RNA binding proteins.

$\mathrm{A}, \mathrm{B}$, and $\mathrm{C}$ indicate structure-based multiple sequence alignments of RNA binding proteins belonged to groups 1,2 , and 4 , respectively. The secondary structures of the base sequence of the each multiple alignment are shown at the top of the alignments.

\subsection{Building profile hidden Markov models}

Profile HMM is a probabilistic model based on a consensus amino acid sequence; it presents profiles from its multiple sequence alignments as a position-specific scoring system suitable for 
searching distant homologous proteins. There are position-specific scores for a distribution of amino acids and the scores for opening and extending a residue insertion or deletion in the position-specific scoring system of profile HMM. This nature of the profile HMM captures valuable information about properties of conserved at positions in the multiple alignments. We built HMMs based on the multiple alignments of RNA binding proteins belonging to each group as shown in Figure 4. HMMs were also built on the basis of the structure-based multiple alignments of non-RNA binding proteins as a control.

To evaluate the HMMs obtained in this study, we tested them by application to the PDB40 sequence dataset [35]. Since a number of domain sequences in the PDB are very similar to others and have redundancy, the sequence database PDB40 included in ASTRAL SCOP 1.63 [35] was used in the present study. The PDB40 has a subset of 5,226 sequences that consists almost entirely of distinct protein domains, derived from the SCOP database to yield the largest set with pairwise sequence identities of $40 \%$ or less [35]. The result indicated that RNA binding proteins with OB-fold (35 proteins) and non-RNA biding proteins with OB-fold listed in Table 2 were selected with the E-values lower than 1.0 by HMMs derived from RNA binding proteins and non-RNA binding proteins, respectively. This result suggests that HMMs obtained by structure-based multiple alignment may extract information to predict RNA binding proteins with OB-fold. It is, however, noted that there are a few false positives: that is, non-RNA binding proteins, such as Bacterioferritin [42] and Vinculin [43] are selected, giving E-values of 5.3 and 1.4, respectively, by using HMMs derived from RNA binding proteins. It is reported that proteins which give an E-value lower than 10.00 are assumed to match up to a HMM [34]. Therefore, we have used two different E-value cutoffs (1.0 and 10.0) to predict RNA binding proteins with OB-fold.

To demonstrate the validity of HMMs derived from the structure-based multiple alignment of RNA binding proteins with OB-fold, the results described above were compared with those obtained by HMMs from sequence-based multiple alignment of OB-fold in the SCOP database. For this purpose, the amino acid sequences of RNA binding proteins with OB-fold in the SCOP database were aligned with ClustalW 1.83 [44], HMMs were built by HMMER as with those derived from the structure-based multiple alignment, and then applied to the PDB40. The analysis showed that 22 RNA binding proteins with OB-fold were selected, which was about $63 \%(22 / 35=$ $0.63)$ compared with those obtained by HMMs derived from the structure-based alignment. The result demonstrates the authenticity of HMMs derived from the structure-based multiple alignment of RNA binding proteins with OB-fold.

Next, we tested them by application to the sequence database of Pyrococcus horikoshii OT3: a hyperthermophilic archaeon whose genome comprises 2061 open reading frames in which 557 proteins are functionally annotated [36]. The prediction using the HMMs showed that 60 proteins gave E-values less than 10.0, of which 13 proteins were suggested to be RNA-binding proteins with OB-fold, giving E-values lower than 1.0 (Table 3). Among 13 proteins, 9 proteins are annotated to be RNA binding proteins, while 4 proteins are hypothetical proteins in the database. It is thus likely that the four hypothetical proteins may be RNA binding proteins. In addition, two methionyl-tRNA synthetases (PH0285, PH0993), isoleucyl-tRNA synthetase (PH1065), ribosomal protein L10E (PH1999), eIF2 gamma subunit (PH1706) and transcriptional regulatory protein hypF (PH0897) which were not included in the OB-fold dataset in SCOP were predicted to be RNA-binding proteins. Although these $P$. horikoshii protein structures have not been determined, the C-terminal domain of methionyl-tRNA synthetase from $P$. abyssi is categorized in OB-fold in the latest version of SCOP [45]. Moreover, the ribosomal protein L10E from H. marismortui is predominantly composed of antiparallel $\beta$-sheets with $\alpha$-helix [18], and the eIF2 gamma subunit from $P$. abyssi is known to be all $\beta$ protein with a Greek Key $\beta$ barrel similar to OB-fold [46]. In contrast, the application of the HMMs derived from the structure-based alignments of non-RNA binding proteins 
to the $P$. horikoshii database predicted 36 proteins to be OB-fold structures with E-value cutoff of 10.0, of which only one RNA binding protein (RNA methyltransferase) was predicted. These results again suggest that HMMs thus obtained may extract information to predict RNA binding proteins with OB-fold.

Finally, we searched RNA binding proteins with OB-fold in the rice full-length cDNA database release on October 2003 [37]. The result showed that 24 proteins, including 10 annotated and 14 hypothetical proteins, were suggested to be RNA binding proteins with OB-fold, yielding E-values less than 1.0 (Table 4). Actually, all 10 annotated proteins are known to be RNA binding proteins. It is suggested that 14 hypothetical proteins may be RNA binding proteins.

Table 2. Classification of OB-fold structures.

\begin{tabular}{|c|c|c|c|}
\hline Group 1 & Group 2 & Group 3 & Group 4 \\
\hline RNA binding Proteins & RNA binding Proteins & non RNA binding Proteins & RNA binding Proteins \\
\hline Initiation factor & Ribosomal Protein & Bacterial Superantigen & tRNA Synthetase \\
\hline 1BKB::75-139 & 1FJF:L:31-105 & 1B1Z:A:28-107 & 1ASZ:A:105-200 \\
\hline 1D7Q:A:41-109 & 1FJF:Q:2-105 & 1BXT:A:30-117 & 1B8A:A:14-100 \\
\hline 2EIF:A:74-132 & $1 \mathrm{JJ} 2: \mathrm{A}: 35-84$ & 1ENF:A:23-105 & 1C0A:A:10-106 \\
\hline Myf domain & 1RL2:A:71-117 & 1ESF:A:31-120 & 1EFW:A:10-104 \\
\hline 1EUJ:A:7-96 & S1 RNA-binding domain & 1ET9:A:18-95 & 1KRS::65-149 \\
\hline 1GD7:A:10-90 & 1HH2:P:133-198 & 1JCK:B:30-121 & 1LYL:A:63-150 \\
\hline 1PYS:B:41-118 & 1K0R:A:110-183 & 1STE::28-120 & \\
\hline Staphylococcal nuclease & 1SRO::1-76 & 3SEB::30-122 & non RNA binding Proteins \\
\hline 1SNC::7-96 & Cold Shock Protein & & ssDNA-binding protein \\
\hline mRNA Capping Enzyme & 1C9O:A:1-66 & & $1 G V P:: 1-65$ \\
\hline 1CKM:A:240-300 & $1 \mathrm{CSP}:: 1-67$ & & 1JE5:A:3-181 \\
\hline RNA polymerase II & 1G6P:A:1-66 & & 1JMC:A:311-403 \\
\hline \multirow[t]{2}{*}{ 1I50:H:2-62 } & 1H95:A:9-76 & & 1KXL:A:23-147 \\
\hline & $1 \mathrm{MJC}:: 2-70$ & & 1OTC:A:51-150 \\
\hline non RNA binding Proteins & Initiation factor & & 1OTC:A:365-495 \\
\hline DNA ligase & 1AH9::6-70 & & 1OTC:B:37-129 \\
\hline 1DGS:A:315-392 & 1JJG:A:31-102 & & 1PFS:A:1-73 \\
\hline 1FVI:A:190-293 & 1JT8:A:19-86 & & 1QUQ:A:68-151 \\
\hline Tail-associated lysozyme gp5 & 1KL9:A:14-87 & & 1QUQ:B:21-90 \\
\hline 1K28:A:6-129 & 1LUZ:A:10-88 & & 1QVC:A:1-115 \\
\hline ssDNA-binding protein & RNA polymerase II & & 3ULL:A:10-124 \\
\hline $1 \mathrm{GPC}:: 38-195$ & 1GO3:E:80-167 & & DNA helicase \\
\hline 1JMC:A:193-292 & 1I50:H:92-146 & & 1BVS:A:1-64 \\
\hline 1OTC:A:223-300 & RHO termination factor & & 1CUK::1-64 \\
\hline Molybdate-binding protein & $1 \mathrm{~A} 62:: 46-125$ & & RecG \\
\hline 1B9M:A:200-252 & & & 1GM5:A:165-250 \\
\hline 1B9M:A:127-183 & non RNA binding Proteins & & Bacterial AB5 toxins \\
\hline 1FR3:A:1-67 & DNA ligase & & 1EFI:D:12-103 \\
\hline 1G29:1:241-301 & 1A0I::240-331 & & 1PRT:B:99-193 \\
\hline $1 \mathrm{G} 29: 1: 302-358$ & Bacterial Superantigen & & 1PRT:D:1-110 \\
\hline 1GUT:A:2-68 & $1 \mathrm{AN} 8:: 20-95$ & & 1PRT:F:2-99 \\
\hline 1H9M:A:74-141 & 1EU3:A:15-95 & & 1QNU:A:101-169 \\
\hline \multirow[t]{10}{*}{ 1H9M:A:1-73 } & 2QIL:A:15-96 & & 1TII:D:14-98 \\
\hline & Inorganic pyrophosphatase & & 2BOS:A:102-169 \\
\hline & 1E9G:A:90-162 & & 3CHB:D:12-103 \\
\hline & 1I40:A:10-112 & & Superantigen-like Protein \\
\hline & 1QEZ:A:1010-1110 & & 1M4V:A:23-100 \\
\hline & 2PRD::10-112 & & Metalloproteinase Inhibitor \\
\hline & Histidine kinase CheA & & 1BQQ:T:16-109 \\
\hline & 1B3Q:A:561-626 & & 1BR9::15-109 \\
\hline & Laminin-binding domain of & grin & 1UEA:B:15-107 \\
\hline & 1JB3:A:14-110 & & $\begin{array}{l}\text { Chemotaxis protein } \mathrm{CheW} \\
\text { 1K0S:A:30-95 }\end{array}$ \\
\hline
\end{tabular}


Table 3. Predicted RNA binding proteins in $P$. horikoshii OT3.

The database was searched by HMMER with restriction of E-value $<10.0$. Among 31 annotated proteins, 16 proteins with boldface are annotated as RNA binding proteins, while 15 other proteins are indicated by underlines. RNA binding proteins boxed were not used to build the HMMs.

\begin{tabular}{|c|c|c|c|c|c|c|c|c|c|c|c|c|c|c|}
\hline \multicolumn{3}{|l|}{ Group 1} & \multicolumn{7}{|l|}{ Group 2} & \multicolumn{5}{|c|}{ Group 4} \\
\hline Name & Score & E-value & Name & Score & E-value & Name & Score & E-value & Name & Score & E-value & Name & Score & E-value \\
\hline РH0993 & 44.4 & $9.1 \mathrm{E}-11$ & PH0961 & 39.3 & $3.1 \mathrm{E}-09$ & PH0928 & -5.3 & 3.6 & PH1507 & -7.6 & 6.7 & PH1020 & 143.7 & $1.2 \mathrm{E}-40$ \\
\hline PH1381 & 22.4 & 0.00037 & PH1908 & 38.3 & 5.9E-09 & PH1100 & -5.4 & 3.6 & PH1882 & -7.9 & 7.2 & РH0241 & 64.1 & $1 \mathrm{E}-16$ \\
\hline PH0536 & 4.8 & 0.16 & PH1568 & 23.1 & 0.00024 & $\underline{\mathrm{PH} 1647}$ & -5.9 & 4.2 & PH1706 & -8.0 & 7.3 & PH0340 & -6.0 & 0.016 \\
\hline РН0285 & -2.1 & 1.0 & PH1775 & 18.4 & 0.0059 & PH1399 & -6.0 & 4.3 & PH1179 & -8.0 & 7.4 & РH0123 & -27.1 & 1.4 \\
\hline PH1212 & -4.0 & 1.7 & PH0340 & 2.2 & 0.48 & $\underline{\mathrm{PH} 0275}$ & -6.0 & 4.4 & PH1740 & -8.1 & 7.5 & PH0283 & -28.0 & 1.6 \\
\hline PH0117 & -4.2 & 1.8 & PH0043 & 0.3 & 0.79 & $\overline{\mathrm{PH} 0128}$ & -6.6 & 5.1 & PH0988 & -8.1 & 7.6 & $\underline{\mathrm{PH} 0549}$ & -30.5 & 2.8 \\
\hline PH0822 & -5.3 & 2.4 & PH1770 & -1.5 & 1.3 & $\underline{\mathrm{PH} 1907}$ & -6.6 & 5.1 & PH0326 & -8.2 & 7.7 & PH0618 & -33.5 & 5.2 \\
\hline PH1995 & -5.8 & 2.8 & PH0484 & -1.7 & 1.3 & $\overline{\mathrm{PH} 0235}$ & -6.9 & 5.5 & PH0121 & -8.3 & 8.0 & PH0043 & -33.8 & 5.5 \\
\hline PH1740 & -5.9 & 2.8 & PH1065 & -2.3 & 1.6 & PH0346 & -7.0 & 5.6 & PH0863 & -8.9 & 9.4 & PH1897 & -35.2 & 7.4 \\
\hline PH1568 & -6.1 & 3.0 & PH1353 & -3.4 & 2.2 & PH1349 & -7.2 & 6.0 & PH0387 & -9.0 & 9.8 & PH1701 & -35.5 & 7.8 \\
\hline PH1999 & -8.3 & 5.2 & PH0117 & -3.5 & 2.2 & $\underline{\mathrm{PH} 0800}$ & -7.3 & 6.2 & \begin{tabular}{|l|} 
PH0897 \\
\end{tabular} & -9.1 & 9.8 & & & \\
\hline PH1622 & -10.3 & 8.9 & $\underline{\mathrm{PH} 1262}$ & -4.0 & 2.5 & $\overline{\mathrm{PH} 0841}$ & -7.5 & 6.4 & PH1593 & -9.1 & 9.9 & & & \\
\hline & & & PH0650 & -5.1 & 3.4 & $\underline{\mathrm{PH} 0764}$ & -7.5 & 6.5 & & & & & & \\
\hline
\end{tabular}

Table 4. Predicted RNA binding proteins in the rice full-length cDNA database.

The database was searched by HMMER with restriction of E-value $<10.0$. Among 14 annotated proteins, 11 proteins with boldface are annotated as RNA binding proteins, while 3 other proteins are indicated by underlines. RNA binding proteins boxed were not used to build the HMMs.

\begin{tabular}{|c|c|c|c|c|c|c|c|c|c|c|c|}
\hline \multirow{2}{*}{$\frac{\overline{\text { Group } 1}}{\text { Name }}$} & \multirow[b]{2}{*}{ Score } & \multicolumn{2}{|r|}{ Group 2 } & \multirow[b]{2}{*}{ Score } & \multirow[b]{2}{*}{ E-value } & \multirow[b]{2}{*}{ Name } & \multirow[b]{2}{*}{ Score } & \multirow[b]{2}{*}{ E-value } & \multicolumn{3}{|l|}{ Group 4} \\
\hline & & E-value & Name & & & & & & Name & Score & E-value \\
\hline J023012P17 & 43.0 & $2.4 \mathrm{E}-09$ & J013001008 & 44.1 & 1.1E-09 & J023095J08 & 4.2 & 2.9 & 002-160-A04 & 61.6 & $6.1 \mathrm{E}-15$ \\
\hline 002-157-A08 & 40.7 & $1.2 \mathrm{E}-08$ & J023036A08 & 42.5 & 3.4E-09 & J023136G11 & 3.8 & 3.2 & J013071I10 & 58.6 & $5 \mathrm{E}-14$ \\
\hline 002-137-H02 & 36.5 & $2.3 \mathrm{E}-07$ & J013106B18 & 39.2 & 3.4E-08 & J033046J06 & 2.0 & 5.2 & 002-117-F07 & 41.2 & $8.5 \mathrm{E}-09$ \\
\hline \multirow[t]{10}{*}{ 001-036-F11 } & 21.1 & 0.0097 & 002-159-D04 & 35.2 & $5.4 \mathrm{E}-07$ & 001-027-E01 & 0.6 & 7.6 & 002-126-D07 & 32.5 & $3.5 \mathrm{E}-06$ \\
\hline & & & 002-171-F11 & 33.9 & $1.4 \mathrm{E}-06$ & 001-044-D07 & 0.3 & 8.2 & 002-165-A01 & 27.8 & $9.4 \mathrm{E}-05$ \\
\hline & & & $\mathrm{J} 023075 \mathrm{D} 13$ & 31.2 & 8.6E-06 & 002-149-B07 & -0.3 & 9.7 & J023105D18 & 23.2 & 0.00035 \\
\hline & & & J023061H10 & 30.2 & $1.8 \mathrm{E}-05$ & & & & 001-121-B05 & 13.2 & 0.0028 \\
\hline & & & 001-020-G05 & 29.5 & $2.9 \mathrm{E}-05$ & & & & $\mathrm{~J} 033070 \mathrm{O} 21$ & 2.9 & 0.025 \\
\hline & & & \begin{tabular}{|l|}
$\mathrm{J} 023088 \mathrm{~B} 11$ \\
\end{tabular} & 27.8 & $9.3 \mathrm{E}-05$ & & & & $\underline{\mathrm{J} 023027 \mathrm{~K} 10}$ & 15.2 & 1.1 \\
\hline & & & 001-203-D03 & 24.3 & 0.001 & & & & $\mathrm{~J} 023123 \mathrm{~B} 03$ & 22.2 & 5.0 \\
\hline & & & J033149P22 & 22.4 & 0.0039 & & & & $\underline{\mathrm{J} 013157 \mathrm{O} 09}$ & 24.7 & 8.4 \\
\hline & & & $\mathrm{J} 013110 \mathrm{C} 21$ & 14.2 & 0.19 & & & & J023001J17 & 24.9 & 8.8 \\
\hline & & & $\mathrm{J} 013106 \mathrm{~A} 17$ & 5.9 & 1.8 & & & & J013146B15 & 25.4 & 9.7 \\
\hline
\end{tabular}

\subsection{In conclusion}

We are currently concerned with structural genomics project on proteins involved in transcription and translation. One major motivation of structural genomics projects is that the determination of the structure of a protein provides insight into its molecular function, which is a step toward understanding its cellular function. It is now common knowledge that RNA molecules play essential roles in transcription and translation. Usually, RNA molecules perform these functions in close association with RNA binding proteins. Hence, there is a pressing requirement for computational methods for prediction of RNA binding proteins in vast amounts of genome sequence information. Recently, several groups have developed algorithms to predict protein function often employing either sequence-based or structure-based comparisons. However, the methods specific for a given function or fold are limited. The result obtained in this study demonstrates feasibility of HMMs derived from structure-based multiple alignments for prediction 
of RNA binding proteins with OB-fold. Although OB-fold selected in the present study is a characteristic fold, predominantly comprising of $\beta$-strands, the strategy presented in this paper can be in principle applicable to any other structural motifs found in RNA binding proteins. Since some of the motifs have no specific biological function alone but are part of larger structural and functional assembles, the present method would provide clues as to protein functions of unannotated proteins and also be useful for a target selection for structural genomics.

This work was supported in part by a grant of Rice Genome Project PR-3007, MAFF, Japan and a grant from the National Project on Protein Structural and Functional Analyses, Japan.

\section{References}

[1] H.W. Mewes, K. Albermann, K. Heumann, S. Liebl, F. Pfeiffer, Nucleic Acids Res., 25, 28-39 (1997).

[2] E. Pennisi, Science, 279, 978-979 (1998).

[3] T.I. Zarembinski, L.W. Hung, H.J. Mueller-Dieckmann, K.K. Kim, H. Yokota, R. Kim, S.H. Kim, Proc. Natl. Acad. Sci. USA., 95, 15189-15193 (1998).

[4] G.T. Montelione, S. Anderson, Nature Struct. Biol., 6, 11-12 (1999).

[5] D. Christendat, A.Yee, A. Dharamsi, Y. Kluger, A. Savchenko, J.R. Cort, V. Booth, C.D. Mackereth, V. Saridakis, I. Ekiel, G. Kozlov, M.A. Kennedy, A.R. Davidson, F.F. Pai, M. Gerstein, A.M. Edwards, C.H. Arrowsmith, Nature Struct. Biol., 7, 903-909 (2000).

[6] M.B. Eisen, P.T. Spellman, P.O. Brown, D. Bostein, Proc. Natl. Acad. Sci. USA., 95, 14863-14868 (1998).

[7] M. Brown, W.N. Grundy, D. Lin, N. Cristianini, C.W. Sugnet, T.S. Furey, M.Jr. Ares, D. Houssler, Proc. Natl. Acad. Sci. USA., 97, 262-267 (2000).

[8] C.A. Wilson, J. Kreychman, M. Gerstein, J. Mol. Biol., 297, 233-249 (2000).

[9] D. Devos, A Valencia, Proteins, 41, 98-107 (2000).

[10] B. Rost, J. Mol. Biol., 318, 595-608 (2002).

[11] D.B. Kell, R.D. King, Trends Biotechnol., 18, 93-98 (2000).

[12] R.D. King, A. Karwath, A. Clare, L. Dehaspe, Bioinformatics, 17, 445-454 (2001).

[13] A. Clare, R.D. King, Bioinformatics, 18, 160-166 (2002).

[14] A.R. Ortiz, A. Kolinski, P. Rotkiewicz, B. Ilkowski, J. Skolnick, Proteins Suppl, 3,177-185 (1999).

[15] J. Pillardy, C. Czaplewski, A. Liwo, J. Lee, D.R. Ripoll, R. Kazmierkiewicz, S. Oldziej, W.J. Wedemeyer, K.D. Gibson, Y.A. Arnautova, J. Saunders, Y.J. Ye, H.A. Scheraga, Proc. Natl. Acad. Sci. USA., 98, 2329-2333 (2001).

[16] K.T. Simons, I. Ruczinski, C. Kooperberg, B.A. Fox, C. Bystroff, D. Baker, Proteins, 34, 82-95(1999).

[17] R. Bonneau, D. Baker, Annu. Rev. Biophys. Biomol. Struct., 30, 173-189 (2001).

[18] A. Bateman, L. Coin, R. Darbin, R.D. Finn, V. Hollich, S. Griffiths-Jones, A. Khanna, M. Marshall, S. Moxon, E.L. Sonnhammer, et al., Nucleic Acids Res., Database issue: D138-D141(2004).

[19] R. Bonneau, N.S. Baliga, E.W. Deutsch, P. Shannon, L. Hood, Genome Biology, 5, R52 1-15 (2004).

[20] C.B. Burge, T. Tuschl, P.A. Sharp, Splicing of precursors to mRNAs by the spliceosomes. In The RNA word, $2^{\text {nd }}$ ed., Gesteland, R.F., Cech, T.R., and Atkins, J.E. eds. Cold Spring Harbor, New York: Cold Spring Harbor Laboratory Press pp 525-560 (1999). 
[21] N. Ban, P. Nissen, J. Hansen, P.B. Moore, T.A. Steitz, Science, 289, 905-920 (2000).

[22] C.G. Burd, G. Dreyfuss, Science, 265, 615-621 (1994).

[23] V. Ramakrishnan, S.W. White, Trend Biochem. Sci., 23, 208-212 (1998).

[24] A. Nakagawa, T. Nakashima, M. Taniguchi, H. Hosaka, M. Kimura, I. Tanaka, EMBO J., 18, 1459-1467 (1999).

[25] T. Nakashima, M. Yao, S. Kawamura, K. Iwasaki, M. Kimura, I. Tanaka, RNA, 7, 692-701 (2001).

[26] A.G. Murzin S.E. Brenner, T. Hubbard, C. Chothia, J. Mol. Biol., 247, 536-540 (1995).

[27] E.E. Abola, J.L. Sussman, J. Prilusky, N.O. Manning, Methods Enzymol., 276, 556-571 (1997).

[28] W. Kabsch, C. Sander, Biopolymers, 22, 2577-637 (1983).

[29] L. Holm, C. Sander, J. Mol. Biol., 233, 123-138 (1993).

[30] R. William, R. Taylor, A. Christine, A. Orengo, J. Mol. Biol., 208, 1-22 (1988).

[31] J.H. Ward, J Am Stat Assoc., 58, 236-244 (1963).

[32] A. Richard, A. Becker, J.M. Chambers, A.R. Wilks, The New S Language. Chapman \& Hall, New York. (1988).

[33] S.F. Altschul, D.J. Lipman, SIAM J. Appl. Math., 49, 197-209 (1989).

[34] S. Eddy, HMMER: profile HMMs for protein sequence analysis. http://hmmer.wustl.edu/ (2003).

[35] S.E. Brenner, P. Koehl, M. Levitt, Nucleic Acids Res., 28, 254-256 (2000).

[36] Y. Kawarabayashi, et al., DNA Res., 5, 55-76 (1998).

[37] S. Kikuchi, et al., Science, 301, 376-379 (2003).

[38] A.G. Murzin, EMBO J., 12, 861-867 (1993).

[39] P.J. Kraulis, J. Appl. Crystallogr., 24, 946-950 (1991).

[40] E.A. Merrit, M.E.P. Murphy, Acta Crystallogr., D50, 869-873 (1994).

[41] B.L. Golden, D.W. Hoffman, V. Ramakrishnan, S.W. White, Biochemistry, 32, 12812-12820 (1993).

[42] F. Frolow, A.J. Kalb, J. Yariv, Nat.Struct. Biol., 1, 453-460 (1994).

[43] C. Bakolitsa, J.M. de Pereda, C.R. Bagshaw, D.R. Critchley, R.C. Liddington, Cell, 99, 603-613 (1999).

[44] J.D. Thompson, D.G. Higgins, T.J. Gibson, Nucleic Acids Res., 22, 4673-4680 (1994).

[45] T. Crepin, E. Schmitt, S. Blanquet, Y. Mechnulam, Biochemistry, 43, 2635-2644 (2004).

[46] E. Schmitt, S. Blanquet, Y. Mechulam, EMBO J., 21, 1821-1832 (2002). 